\title{
Solid-State NMR Spectroscopy of Molecular Hydrogen Trapped Inside an Open-Cage Fullerene
}

\author{
Marina Carravetta, ${ }^{\dagger}$ Yasujiro Murata, ${ }^{\ddagger}$ Michihisa Murata, ${ }^{\ddagger}$ Ivo Heinmaa,, Raivo Stern,, \\ Ana Tontcheva," Ago Samoson, $\S^{\S}$ Yves Rubin," Koichi Komatsu, ${ }^{\ddagger}$ and Malcolm H. Levitt ${ }^{\star, \dagger}$ \\ School of Chemistry, University of Southampton, SO17 1BJ Southampton, UK, Institute for Chemical Research, \\ Kyoto University, Uji, Kyoto 611-0011, Japan, Institute of Chemical Physics and Biophysics, 12618 Tallinn, \\ Estonia, and Department of Chemistry and Biochemistry, University of California, Los Angeles, \\ Los Angeles, California 90095-1569
}

Received December 5, 2003; E-mail: mhl@soton.ac.uk

The trapping of atoms and molecules ${ }^{1-4}$ inside fullerene cages provides a set of remarkable systems for exploring atomic interactions and molecular dynamics. ${ }^{5}$ Recently, Murata and co-workers ${ }^{4,6}$ have achieved a very high-yield insertion of molecular hydrogen into the azo-thia-open-cage fullerene $\left(\mathrm{H}_{2} @ \mathrm{ATOCF}\right),{ }^{4}$ shown in Figure 1.

In this article we describe ${ }^{1} \mathrm{H}$ NMR measurements on the endohedral $\mathrm{H}_{2}$ molecules of $\mathrm{H}_{2} @$ ATOCF in the solid state. We observe a small anisotropy of the $\mathrm{H}_{2}$ rotation inside the fullerene cage and estimate the correlation time of the $\mathrm{H}_{2}$ rotation. These studies complement the low-temperature NMR studies of $\mathrm{H}_{2}$ molecules trapped in the interstices of solid $\mathrm{C}_{60} \cdot{ }^{7}$

Experimental ${ }^{1} \mathrm{H}$ spectra for $\mathrm{H}_{2} @$ ATOCF obtained by magicangle spinning (MAS) solid-state NMR are shown in Figure 2. Figure $2 \mathrm{a}$ shows the broad ${ }^{1} \mathrm{H}$ spectrum in the absence of sample rotation. At a slow MAS frequency, a set of narrow sidebands appears (Figure 2b). As the spinning frequency is increased (Figure $2 \mathrm{c}-\mathrm{d})$, the sidebands spread out and the centerband grows larger. The narrow centerband has a line width of $0.56 \mathrm{ppm}$ and an isotropic chemical shift of $-7.5 \mathrm{ppm}$ with respect to TMS. This unusual shift assigns the narrow peak to the endohedral $\mathrm{H}_{2}$ and is consistent with solution NMR on the same species. ${ }^{4}$ The broad feature is attributed to the relatively immobile exohedral protons and to the proton impurities in the Teflon probe parts. The dipole-dipole couplings involving the endohedral protons are averaged to a large extent by the rapid motion of the $\mathrm{H}_{2}$ molecule inside the cavity. The residual dipolar couplings are eliminated by the magic-angle rotation, leading to very narrow ${ }^{1} \mathrm{H}$ signals from the endohedral $\mathrm{H}_{2}$. Similar behavior is observed for other solids displaying high molecular mobility. ${ }^{8-11}$

If the rotational motion in the cavity is assumed to be uniaxial, the motionally averaged dipole-dipole between the $\mathrm{H}_{2}$ nuclei coupling may be written as

$$
b_{\mathrm{HH}}^{\mathrm{eff}}=b_{\mathrm{HH}}^{\text {static }} S, \quad S \in[0,1]
$$

where $S$ is the second-rank order parameter $S=$ $\left\langle\left(3 \cos ^{2} \theta-1\right) / 2\right\rangle$, and $\theta$ is the angle between the $\mathrm{HH}$ vector and a molecule-fixed rotational order axis. The angular brackets represent a time or ensemble average. $S=0$ corresponds to isotropic rotation, while $S=1$ corresponds to the static case. The dipole-

University of Southampton.

\#yoto University.

$\S$ Institute of Chemical Physics and Biophysics, Tallinn.

" University of California, Los Angeles.

4092 - J. AM. CHEM. SOC. 2004, 126, 4092-4093

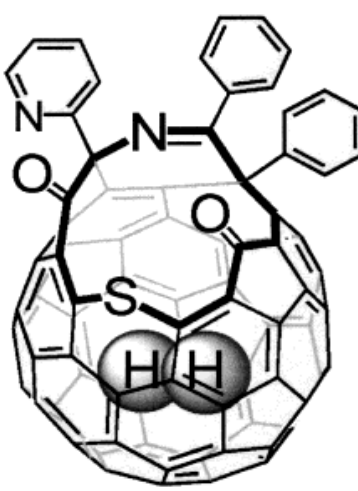

Figure 1. Molecular structure of $\mathrm{H}_{2} @$ ATOCF.

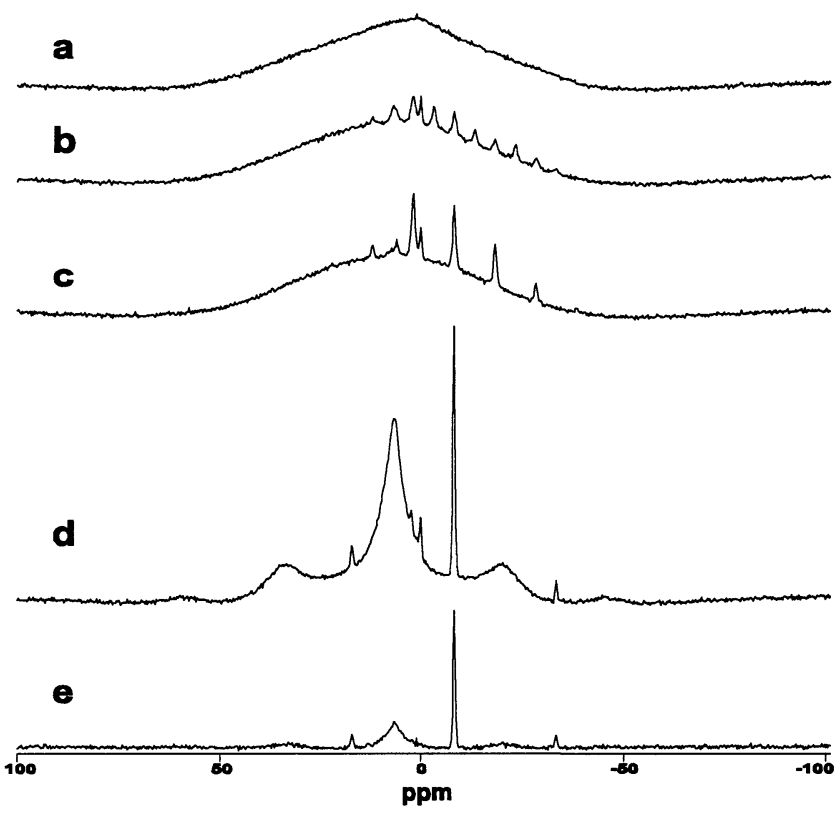

Figure 2. ${ }^{1} \mathrm{H}$ spectra of a powder sample of $\mathrm{H}_{2} @ \mathrm{ATOCF}$ at a field of 9.4 $\mathrm{T}$. The spectra in a-d were acquired using a simple $90^{\circ}$ pulse to excite transverse ${ }^{1} \mathrm{H}$ magnetization. (a) No sample rotation, (b) MAS at $2.0 \mathrm{kHz}$ (c) MAS at $4.0 \mathrm{kHz}$, (d) MAS at $10.0 \mathrm{kHz}$, and (e) DQ-filtered spectrum recorded at $10.0 \mathrm{kHz}$ MAS using the pulse sequence in Figure 3.

dipole coupling in the absence of motion is

$$
b_{\mathrm{HH}}^{\text {static }}=-\left(\mu_{0} / 4 \pi\right) \gamma_{\mathrm{H}}{ }^{2} \hbar r_{\mathrm{HH}}{ }^{-3}
$$

where $r_{\mathrm{HH}}$ is the proton-proton bond length and $\gamma_{\mathrm{H}}$ is the proton 10.1021/ja031536y CCC: $\$ 27.50$ @ 2004 American Chemical Society 


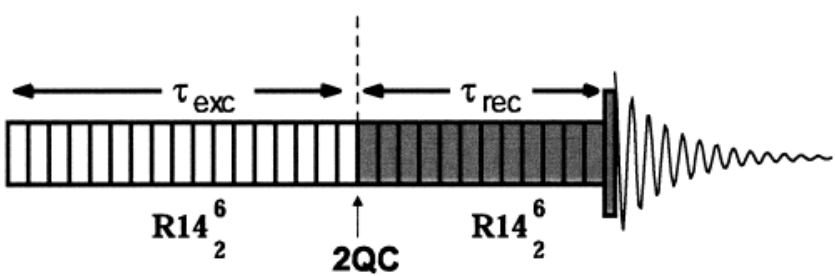

Figure 3. Rf pulse sequence for DQ-filtered ${ }^{1} \mathrm{H}$ NMR in the presence of MAS. Longitudinal ${ }^{1} \mathrm{H}$ magnetization is converted into DQ coherence by a $\mathrm{R} 14_{2}{ }^{6}$ sequence of duration $\tau_{\mathrm{exc}}$ and then reconverted back into $z$ magnetization by a second $\mathrm{R} 14_{2}{ }^{6}$ sequence of duration $\tau_{\text {rec }}$ and observed after a $90^{\circ}$ pulse. The shaded elements are given a four-step phase cycle to suppress signals that did not pass through DQ coherences. The interval $\tau_{\text {exc }}$ is varied to acquire a DQ-filtered signal trajectory.

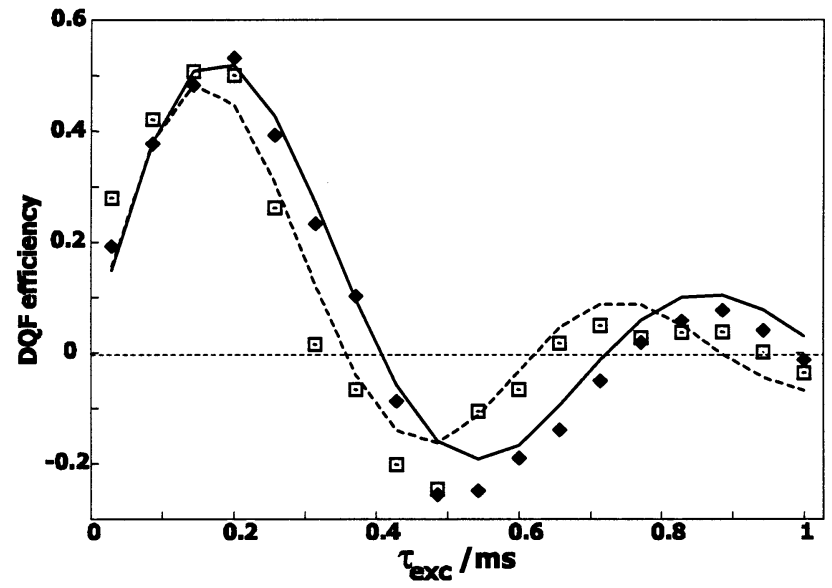

Figure 4. Diamonds: experimental DQ-filtered signal amplitudes at 295 $\mathrm{K}$ as a function of the DQ excitation time $\tau_{\text {exc }}$, with $\tau_{\text {rec }}=200 \mu$ s and a corresponding best fit (solid line) from numerical simulation for a dipoledipole coupling of $-6.1 \mathrm{kHz}$. Squares: experimental DQ-filtered signal amplitudes at $160 \mathrm{~K}$ under the same conditions and a corresponding best fit (dotted line) to a dipole-dipole coupling of $-7.3 \mathrm{kHz}$.

gyromagnetic ratio. This can be evaluated as $b_{\mathrm{HH}}^{\text {static }} / 2 \pi=-296$ $\mathrm{kHz}$ for an internuclear distance of $74 \mathrm{pm}$. The motionally averaged dipolar coupling may be estimated from ${ }^{1} \mathrm{H}-{ }^{1} \mathrm{H}$ double-quantum (DQ) signal trajectories, as performed previously in ${ }^{13} \mathrm{C}$ NMR. ${ }^{12,13}$ The pulse sequence used for the ${ }^{1} \mathrm{H}$ case is shown in Figure 3.

The $\mathrm{R} 14_{2}{ }^{6}$ sequence involves a repetition of the four-pulse element $\left(90_{77.1} 270_{257.1} 90_{-77.1} 270_{-257.1}\right)$, where the rf field amplitude provides a nutation frequency of seven times the MAS frequency. ${ }^{12,13}$ The $\mathrm{rf}$ pulses are defined as $\beta_{\phi}$, where $\beta$ is the flip angle and $\phi$ is the phase, both specified in degrees. This sequence provides efficient DQ homonuclear recoupling, while chemical shifts are decoupled. An experimental DQ-filtered ${ }^{1} \mathrm{H}$ spectrum of $\mathrm{H}_{2} @$ ATOCF at a spinning frequency of $10 \mathrm{kHz}$, using $\tau_{\mathrm{exc}}=\tau_{\mathrm{rec}}$ $=200 \mu \mathrm{s}$ is shown in Figure 2e. The narrow and broad signals both pass through the DQ filter with good efficiency (approximately $53 \%$ for the endohedral $\mathrm{H}_{2}$ signal). The DQ coherences contributing to the sharp signal in Figure 2e involve the pairs of endohedral hydrogens (see Supporting Information).

DQ-filtered signal trajectories obtained at $160 \mathrm{~K}$ and $295 \mathrm{~K}$ by incrementing the $\tau_{\text {exc }}$ interval with $\tau_{\text {rec }}$ fixed at $200 \mu$ s are shown in Figure 4, together with best fits from numerical simulations (see Supporting Information). The best fit simulations deviate at longer recoupling times, probably due to the influence of other protons and to a small asymmetry in the effective dipolar tensor. However, our data do not allow an accurate estimate of the asymmetry parameter. The estimated dipolar coupling varies approximately linearly with temperature, from $\left|b_{\mathrm{HH}}^{\text {eff }} / 2 \pi\right|=7.3 \pm 0.25 \mathrm{kHz}$ at 160 $\mathrm{K}$ to $6.1 \pm 0.2 \mathrm{kHz}$ at $295 \mathrm{~K}$ (the sign cannot be determined). These results show that the motional anisotropy of $\mathrm{H}_{2}$ inside the cavity is very small, with order parameters varying from $|S|=2.3$ $\pm 0.3 \%$ at $160 \mathrm{~K}$ to $|S|=2.0 \pm 0.3 \%$ at $295 \mathrm{~K}$. This suggests that the orifice only slightly perturbs the rotational motion of the endohedral $\mathrm{H}_{2}$.

Spin-lattice relaxation time constants $\left(T_{1}\right)$ were obtained as a function of temperature and spinning frequency. At $295 \mathrm{~K}$, the $T_{1}$ of the endohedral hydrogen peak was observed to be $1.56 \pm$ $0.95 \mathrm{~s}$ at a spinning frequency of $7 \mathrm{kHz}$ and $0.16 \pm 0.01 \mathrm{~s}$ at a spinning frequency of $20 \mathrm{kHz}$, with a rapid variation of $T_{1}$ in the region of $12 \mathrm{kHz}$ spinning frequency (see Supporting Information). We attribute the observed behavior to strong dipole-dipole contact between the endohedral hydrogens and the slowly relaxing exohedral protons at lower spinning speeds and to efficient decoupling of these two reservoirs at higher spinning frequencies. Similar behavior has been observed in other systems. ${ }^{14,15}$ The high spinning frequency data were used as a measure of the endohedral $\mathrm{H}_{2}$ relaxation.

The $T_{1}$ of the endohedral $\mathrm{H}_{2}$ peak at high spinning frequency had an approximately linear dependence upon temperature, varying from $24 \pm 1 \mathrm{~ms}$ at $120 \mathrm{~K}$ to $160 \pm 8 \mathrm{~ms}$ at $295 \mathrm{~K}$. Abragam ${ }^{16}$ has given expressions for the spin-lattice relaxation of hydrogen molecules, assuming that the relaxation is dominated by modulation of the homonuclear dipole-dipole coupling and spin-rotation interaction by rotational diffusion. Our data are consistent with a rotational correlation time for $\mathrm{H}_{2}$ inside the cavity of $\tau_{\mathrm{c}}=2.3 \mathrm{ps}$ at $295 \mathrm{~K}$ and $\tau_{\mathrm{c}}=15.3 \mathrm{ps}$ at $119 \mathrm{~K}$, with a linear dependence in between. A more thorough analysis will be presented elsewhere.

Acknowledgment. This research was supported by the EPSRC (UK), the Estonian Science Foundation, and NHFML (USA).

Supporting Information Available: Experimental and simulation details, two-dimensional double-quantum spectrum, and $T_{1}$ dependence upon spinning frequency (PDF). This material is available free of charge via the Internet at http://pubs.acs.org.

\section{References}

(1) Saunders: M.; Cross, R. J.; Jiménez-Vázquez, H. A.; Shimshi, R.; Khong, A. Science 1996, 271, 1693-1697.

(2) Murphy, T. A.; Pawlic, T.; Weidinger, A.; Höhne, M.; Alcala, R.; Spaeth, J.-M. Phys. Rev. Lett. 1996, 77, 1075-1078.

(3) Rubin, Y.; Jarrosson, T.; Wang, G.-W.; Bartberger, M. D.; Houk, K. N.; Schick, G.; Saunders, M.; Cross, R. J. Angew. Chem., Int. Ed. 2001, 40 1543.

(4) Murata, Y.; Murata, M.; Komatsu, K. J. Am. Chem. Soc. 2003, 125, 7152.

(5) Cross, R. J. J. Phys. Chem. A 2001, 105, 6943.

(6) Murata, Y.; Murata, M.; Komatsu, K. Chem. Eur. J. 2003, 9, 1600-1609.

(7) Tomaselli, M.; Meier, B. H. J. Chem. Phys. 2001, 115, 11017.

(8) Sekine, S.; Kubo, A.; Sano, H. Chem. Phys. Lett. 1990, 171, 155

(9) Shen, Y.; Wu, T.; Yang, Y. Chem. Phys. Lett. 1995, 242, 83-88

(10) Meier, B. H.; Earl, W. L. J. Chem. Phys. 1986, 85, 4905-4911.

(11) Davis, J. H.; Auger, M.; Hodges, R. S. Biophys. J. 1995, 69, 1917-1932.

(12) Carravetta, M.; Edén, M.; Johannessen, O. G.; Luthman, H.; Verdegem, P. J. E.; Lugtenburg, J.; Sebald, A.; Levitt, M. H. J. Am. Chem. Soc. 2001, 123, 10628-10638.

(13) Carravetta, M.; Edén, M.; Brinkmann, A.; Zhao, X.; Levitt, M. H. Chem Phys. Lett. 2000, 321, 205-215.

(14) Andrew, E. R.; Bradbury, A.; Eades, R. G.; Wynn, V. T. Phys. Lett. 1963 4, 99-100.

(15) Gil, A. M.; Alberti, E. Solid State Nucl. Magn. Reson. 1998, 11, $203-$ 209.

(16) Abragam, A. Principles of Nuclear Magnetism; Oxford University Press: 1978, pp 316-321.

JA031536Y 